\title{
Salidroside blocks the proliferation of pulmonary artery smooth muscle cells induced by platelet-derived growth factor-BB
}

\author{
CHANGGUI CHEN ${ }^{1,2}$, YANHONG TANG ${ }^{1,2}$, WEI DENG $^{1,2}$, CONGXIN HUANG $^{1,2}$ and TIANYI WU ${ }^{1-3}$ \\ ${ }^{1}$ Department of Cardiology, Renmin Hospital of Wuhan University; ${ }^{2}$ Cardiovascular Research Institute of Wuhan University, \\ Wuhan, Hubei 430060; ${ }^{3}$ High Altitude Medical Research Institute, Xining, Qinghai 810012, P.R. China
}

Received September 25, 2013; Accepted March 21, 2014

DOI: $10.3892 / \mathrm{mmr} .2014 .2238$

\begin{abstract}
The proliferation of pulmonary artery smooth muscle cells (PASMCs) contributes to the development of pulmonary vascular remodeling, ultimately leading to pulmonary hypertension. In this study, the effects and molecular mechanisms of salidroside on the platelet-derived growth factor (PDGF)-BB-induced proliferation of primary cultured rat PASMCs were investigated. The presented data demonstrated that salidroside significantly inhibited the proliferation and DNA synthesis of PASMCs induced by PDGF-BB in a dose- and time-dependent manner, without cell cytotoxicity. In accordance with these findings, salidroside blocked progression through G0/G1 to $\mathrm{S}$ phase of the cell cycle. The salidroside-induced inhibition of the cell cycle was associated with the inhibition of cyclin D1, cyclin E, cyclin-dependent kinase 2 (CDK2) and CDK4 mRNA expression, as well as an increase in the mRNA expression of p27 in PDGF-BB-stimulated PASMCs. Further experiments showed that the beneficial effect of salidroside on blocking the proliferation of PASMCs was associated with the suppression of the AKT/glycogen synthase kinase $3 \beta$ (GSK3 3 ) signaling pathway, but did not involve the extracellular signal-regulated kinase $1 / 2$, p38 and c-Jun-N-terminal kinase signaling pathways. These results indicate that salidroside suppresses PDGF-BB-induced PASMC proliferation through the AKT/GSK3 $\beta$ signaling pathway and suggests that it may be a feasible therapy for pulmonary vascular remodeling diseases.
\end{abstract}

\section{Introduction}

Pulmonary arterial hypertension (PAH) is characterized by vascular remodeling and a progressive increase in pulmonary vascular resistance, which ultimately leads to right ventricular

Correspondence to: Professor Tianyi Wu, Department of Cardiology, Renmin Hospital of Wuhan University, 238 Jiefang Road, Wuhan, Hubei 430060, P.R. China

E-mail: wutianyiqh@hotmail.com

Key words: salidroside, proliferation, pulmonary artery smooth muscle cells, platelet-derived growth factor-BB failure and death (1). The abnormal proliferation of pulmonary artery smooth muscle cells (PASMCs) is an important feature of PAH that contributes to vascular remodeling and leads to vascular occlusion (2). It is therefore important to clarify the specific molecular mechanisms and signaling pathways that lead to the proliferation of PASMCs. Numerous studies have demonstrated that the pathological proliferation of PASMCs is associated with mitogen-activated protein kinase (MAPK) and AKT signaling pathways $(3,4)$.

The platelet-derived growth factor (PDGF) signaling system consists of four ligands, PDGF-A, PDGF-B, PDGF-C and PDGF-D, and two types of receptor, PDGF $\alpha$-receptor (PDGFR- $\alpha$ ) and $\beta$-receptor (PDGFR- $\beta$ ) (5). PDGF-BB induces the proliferation of vascular smooth muscle cells (VSMCs) and has been proposed to function in the development of atherosclerosis, lung fibrosis, PAH and chronic thromboembolic pulmonary hypertension (6-8). Furthermore, the levels of PDGF in the blood and lung tissues of patients with PAH are increased, further suggesting that PDGF plays a critical role in the development of pulmonary vascular remodeling and the increase in pulmonary arterial pressure (9).

Rhodiola is a widely used medicinal plant that is grown at high altitudes and has a long history of use by Tibetans to enhance the resistance of the body to fatigue. Studies have shown that Rhodiola has various pharmacological properties and exerts anti-inflammatory, anti-anoxia, anti-oxidation, anti-aging, anti-cancer and liver-protective effects (10-14). Salidroside (2-(4-hydroxyphenyl)ethyl $\beta$-D-glucopyranosidee) is one of the major bioactive components extracted from Rhodiola. In lipopolysaccharide (LPS)-induced mastitis, salidroside has been shown to inhibit the extracellular signal-regulated kinase (ERK), p38 and c-Jun N-terminal kinase (JNK) signaling pathways to inhibit inflammation (15). Salidroside can also inhibit the reactive oxygen species-protein kinase C-ERK1/2 signaling pathway, decreasing the proliferation of HT1080 human fibrosarcoma cells (16). Furthermore, salidroside inhibits the proliferation of mesangial cells, induced by high glucose levels, by blocking the ERK1/2 signaling pathway (17). MCF-7 human breast cancer cells can be arrested in G0/G1 phase by salidroside (18). However, the effects of salidroside on the proliferation of PASMCs and its associated mechanisms remain unclear. This study aimed to determine whether salidroside could inhibit the PDGF-BB-induced proliferation of PASMCs, as well as to 
identify the molecular mechanisms that may be responsible for the protective effects of salidroside on PAH.

\section{Materials and methods}

Materials. Salidroside (98\% purity as determined by high-performance liquid chromatography analysis) was ordered from Shanghai Medical Technology Development Co., Ltd. (Shanghai, China). Recombinant human PDGF-BB was ordered from PeproTech (Rock Hill, NJ, USA). Cell counting kit-8 (CCK-8) was obtained from Dojindo Molecular Technologies Inc. (Kumamoto, Japan). A cell proliferation ELISA, BrdU (colorimetric) kit was purchased from Roche (Roche Diagnostics, Mannheim, Germany). TRIzol ${ }^{\circledR}$ was purchased from Invitrogen Life Technologies (Carlsbad, CA, USA). The antibodies used to recognize the total and phosphorylated forms of ERK1/2, p38, JNK, AKT, glycogen synthase kinase $3 \beta($ GSK $3 \beta)$ and GAPDH were ordered from Cell Signaling Technology Inc. (Danvers, MA, USA). Sprague Dawley rats (150-200 g) were ordered from the Wuhan University Center for Animal Experiment (Wuhan, China). All of the animal experiments were approved by the Institutional Animal Care and Use Committee at Renmin Hospital, Wuhan University (Wuhan, China). For the in vitro study, salidroside was dissolved in double-distilled water.

Collagenase I (0.2\%) digestion of PASMCs and cell culture. Sprague Dawley rats (150-200 g) received intraperitoneal anesthesia with $50 \mathrm{mg} / \mathrm{kg} 1 \%$ sodium pentobarbital. The pleura of each rat was then rapidly sectioned, and the heart and lung were removed and placed in a petri dish filled with phosphate-buffered saline to clean the residual blood in a ultra-clean platform. To separate the pulmonary artery, the outer fibrous arterial connective tissue was stripped under a microscope using tweezers and rinsed several times in Dulbecco's modified Eagle's medium/F12 (DMEM/F12) containing $1 \%$ penicillin-streptomycin. The artery was subsequently cut into $1-\mathrm{mm}$ pieces and placed in a centrifuge tube pre-filled with $0.2 \%$ collagenase I, and the tube was then placed in a $\mathrm{CO}_{2}$ incubator to help remove digestive juices. Once every 20 to $30 \mathrm{~min}$, the mixture was observed and gently agitated. The total duration of the digestion was 2-4 h. Following arterial fragment digestion, the cells were centrifuged at $195 \mathrm{x}$ g for $5 \mathrm{~min}$, and the supernatant was discarded. The pellet was rinsed with DMEM/F12 containing $20 \%$ fetal bovine serum and placed in a $37^{\circ} \mathrm{C}, 5 \% \mathrm{CO}_{2}$ incubator for culturing. Four to five days later, the cells were passaged and grown in DMEM/F12 containing $10 \%$ fetal bovine serum. The purity of the PASMC cultures was assessed by the immunocytochemical localization of $\alpha$-smooth muscle actin.

The cells used in this study were taken between passages four and 10. The PASMCs were grown to $70-80 \%$ confluency and then subjected to serum starvation for $24 \mathrm{~h}$ before being used for the experiments. Cells were pretreated with different concentrations of salidroside for $1 \mathrm{~h}$ prior to stimulation with PDGF-BB (20 ng/ml).

Measurement of cell proliferation and DNA synthesis. Cell proliferation was determined by CCK-8 assay according to the manufacturer's instructions (Dojindo Molecular
Technologies Inc.). PASMCs $\left(5 \times 10^{3} /\right.$ well) were grown to $70-80 \%$ confluency in $96-w e l l$ plates and their growth was arrested by serum starvation for $24 \mathrm{~h}$. Cells were subsequently preincubated with various concentrations of salidroside for $1 \mathrm{~h}$ and then treated with PDGF-BB (20 ng/ml) for 12, 24 and $48 \mathrm{~h}$ in the presence of salidroside, prior to being loaded with CCK-8 solution for the final $3 \mathrm{~h}$. Cell proliferation was determined by measuring the optical density at $450 \mathrm{~nm}$. BrdU labeling mixture was added to each well and incubated for $2 \mathrm{~h}$. DNA synthesis was then measured by assessing the incorporation of BrdU using a cell proliferation ELISA kit.

Evaluation of cell viability. The toxicity of salidroside on PASMCs was determined by the trypan blue exclusion test. After 12, 24 and $48 \mathrm{~h}$ of incubation with salidroside at concentrations between 12.5 and $100 \mu \mathrm{M}$, the PASMCs were removed from culture and the cells that excluded $0.4 \%$ trypan blue were counted using an automated cell counter (Invitrogen Life Technologies).

Cell cycle progression assays. Cell cycle progression was determined using a cell cycle and apoptosis analysis kit (Beyotime Institute of Biotechnology, Haimen, China), in accordance with the manufacturer's instructions, and fluorescence-activated cell sorting. Upon reaching 70-80\% confluency in the six-well plates, the PASMCs were subjected to serum starvation for $24 \mathrm{~h}$. The cells were then preincubated with salidroside $(100 \mu \mathrm{M})$ for $1 \mathrm{~h}$ and subsequently treated with PDGF-BB $(20 \mathrm{ng} / \mathrm{ml})$ for $24 \mathrm{~h}$ prior to analysis.

Quantitative polymerase chain reaction ( $q P C R)$. Following serum starvation for $24 \mathrm{~h}$ and preincubation with $100 \mu \mathrm{M}$ salidroside for $1 \mathrm{~h}$, the cells were treated with PDGF-BB for $24 \mathrm{~h}$ in the absence or presence of salidroside. Total RNA was extracted from PASMCs using TRIZol and reverse transcribed into DNA using oligo (dT) primers with the LightCycler 480 SYBR Green 1 Master mix and the LightCycler 480 qPCR system (both Roche Diagnostics). Target gene mRNA expression was normalized to the internal control GAPDH and was expressed relative to the control group. The primer sequences used were as follows: Cyclin D1, forward 5'-GAGACCATCCCCCTGACGGC-3' and reverse 5'-TCTTCCTCCTCCTCGGCGGC-3'; Cyclin E, forward 5'-GTCCTGGCTGAATGTATACATGC-3' and reverse 5'-CCCTATTTTGTTCAGACAACATGGC-3'; CDK2, forward 5'-GCTTTCTGCCATTCTCATCG-3' and reverse 5'-GTCCCCAGAGTCCGAAAGAT-3'; CDK4, forward 5'-ATGTTGTCCGGCTGATGG-3' and reverse 5'-CACCAGGGTTACCTTGATCTCC-3'; P27, forward 5'-TGCAACCGACGATTCTTCTACTCAA-3' and reverse 5'-CAAGCAGTGATGTATCTGATAAACAAGGA-3'; GAPDH, forward 5'-ATTCCATGGCACCGTCAAGG-3' and reverse 5'-AATTCGTTGTCATACCAGGA-3'.

Western blotting. Confluent, serum-starved PASMCs were treated with salidroside $(100 \mu \mathrm{M})$ for $1 \mathrm{~h}$ following exposure to $20 \mathrm{ng} / \mathrm{ml}$ PDGF-BB for the indicated time. The cells were lysed in radioimmunoprecipitation assay buffer containing a protease and phosphatase inhibitor cocktail; the cells were then scraped into 1.5-ml centrifuge tubes. The cell suspension 
was centrifuged at $3,362 \times \mathrm{g}$ for $30 \mathrm{~min}$ at $4^{\circ} \mathrm{C}$, and the protein concentration was assessed by the bicinchoninic acid assay. A total of $20 \mu \mathrm{g}$ protein extract was used for SDS-PAGE, blotted onto Immobilon-FL transfer membranes (Millipore, Billerica, MA, USA) and probed with the relevant antibodies. The protein expression was quantified using the Odyssey infrared imaging system (Li-Cor Biosciences, Lincoln, NE, USA) and protein expression levels were normalized to the GAPDH internal control in the total cell lysate.

Statistical analysis. The results are expressed as the mean \pm standard deviation. Differences among groups were tested by one-way analysis of variance or unpaired two-tailed-tests. $\mathrm{P}<0.05$ was considered to indicate a statistically significant difference.

\section{Results}

Salidroside inhibits the proliferation and DNA synthesis of $P A S M C s$ induced by PDGF-BB. The abnormal proliferation of PASMCs contributes to vascular lesion formation (2). Salidroside is a proven antitumor compound that leads to the suppression of cancer cell growth (18); however, whether salidroside suppresses the growth of PASMC is currently unknown. To determine the effect of salidroside on PASMC proliferation, the effect of different doses of salidroside (12.5-100 $\mu \mathrm{M})$ on proliferation in 12, 24 and $48 \mathrm{~h}$ was investigated using the CCK- 8 cell proliferation assay. Compared with the control, PDGF-BB induced a time-dependent proliferation of PASMCs that was blocked in a concentration-dependent manner by treatment with salidroside for different lengths of time. The greatest level of suppression of proliferation was induced by salidroside at a concentration of $100 \mu \mathrm{M}$ (Fig. 1A). The inhibitory effects of salidroside on DNA synthesis were next investigated by measuring the incorporation of BrdU. Treatment with PDGF-BB increased DNA synthesis in PASMCs in a time-dependent manner, and salidroside significantly suppressed the increase in DNA synthesis in a dose- and time-dependent manner (Fig. 1B).

Different concentrations of salidroside have no effect on PASMC survival. The toxicity of salidroside on PASMCs was determined by the trypan blue exclusion test in the absence or presence of salidroside. As shown in Fig. 2, salidroside concentrations between 12.5 and $100 \mu \mathrm{M}$ did not induce significant levels of cell necrosis in PASMCs after 12, 24 or 48 h compared with untreated cells. Regardless of whether cells were treated with salidroside, cell viability was maintained at $\sim 95 \%$, suggesting that salidroside was not cytotoxic at the tested concentrations.

Salidroside blocks PDGF-BB-induced cell cycle progression through G0/G1- to $S$-phase cell cycle arrest. The effect of salidroside on cell cycle progression was analyzed using flow cytometric analysis. PDGF-BB treatment alone significantly increased the percentage of cells in $\mathrm{S}$ phase whilst decreasing the G0/G1 populations (Fig. 3). By contrast, salidroside-treated cells showed a significant suppression of cell cycle progression. Salidroside at a dose of $100 \mu \mathrm{M}$ reduced the percentage of cells in $\mathrm{S}$ phase and increased the
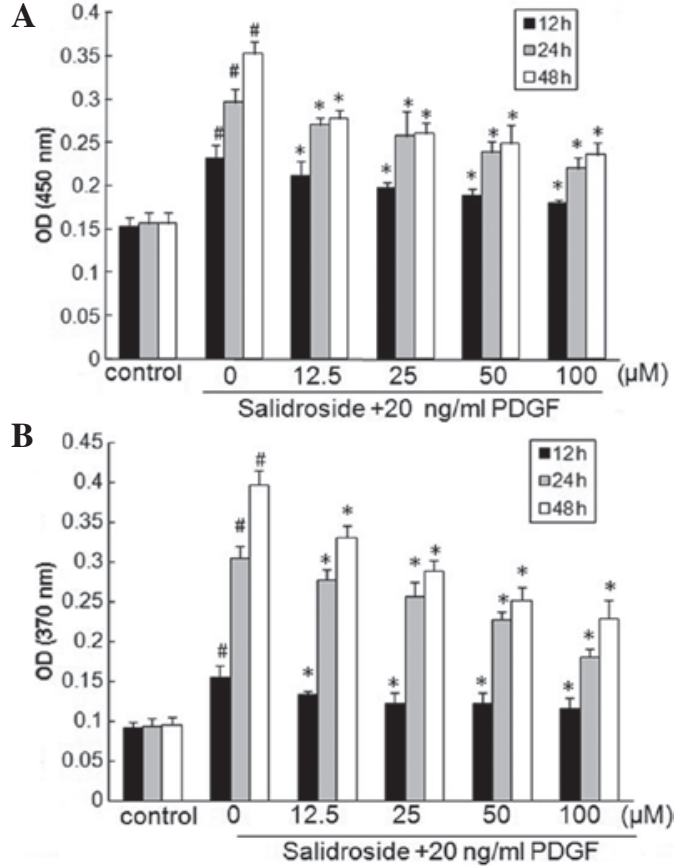

Figure 1. Effects of salidroside on PDGF-BB-induced PASMC proliferation and DNA synthesis. PASMCs were pre-cultured in serum-free medium for $24 \mathrm{~h}$ and then incubated in the absence or presence of salidroside (12.5-100 $\mu \mathrm{M}$ ) with $20 \mathrm{ng} / \mathrm{ml}$ PDGF-BB for a further 12, 24 or $48 \mathrm{~h}$. (A) Cell proliferation was examined by the cell counting kit 8 test. (B) BrdU incorporation was determined with an ELISA-based assay. Data are expressed as the mean $\mathrm{OD} \pm$ standard deviation. ${ }^{\#} \mathrm{P}<0.05$ versus control group; ${ }^{*} \mathrm{P}<0.05$ versus cells exposed to PDGF-BB alone; $\mathrm{n}=5$. OD, optical density; PDGF-BB, platelet-derived growth factor-BB; PASMCs, pulmonary artery smooth muscle cells.

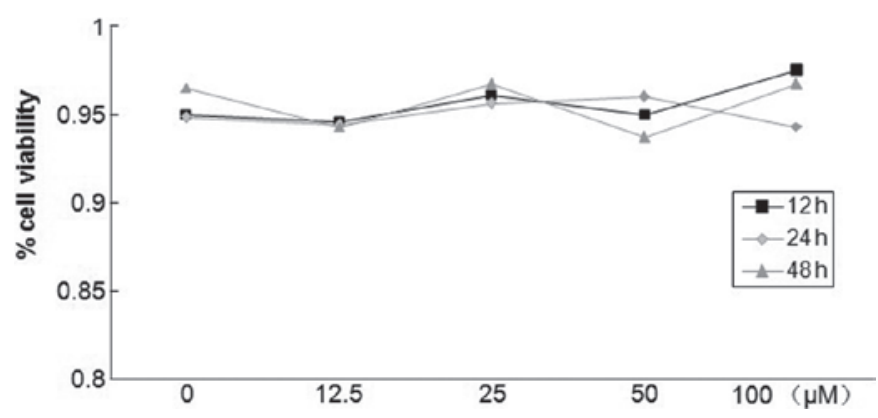

Figure 2. Effect of salidroside on the viability of PASMCs. The viability of PASMCs was evaluated by counting the number of cells that excluded trypan blue in the absence or presence of different concentrations of salidroside for 12,24 or $48 \mathrm{~h}$. $\mathrm{P}>0.05$ versus control group; $\mathrm{n}=4$. PASMCs, pulmonary artery smooth muscle cells.

G0/G1 populations among the PDGF-BB-stimulated cells. This suggests that salidroside affects the G0/G1- to S-phase transition rather than being involved in the $\mathrm{S}$ or $\mathrm{G} 2 / \mathrm{M}$ phases (Fig. 3).

Salidroside downregulates the mRNA expression of cyclin D1, cyclin E, cyclin-dependent kinase 2 (CDK2) and CDK4, and upregulates p $27 m R N A$ expression. To explore the potential mechanisms by which salidroside influences the cell cycle of PASMCs, the mRNA levels of cell cycle regulatory genes, including cyclins, the CDKs and cell cycle inhibitory genes, were examined. PDGF induction significantly increased 
A
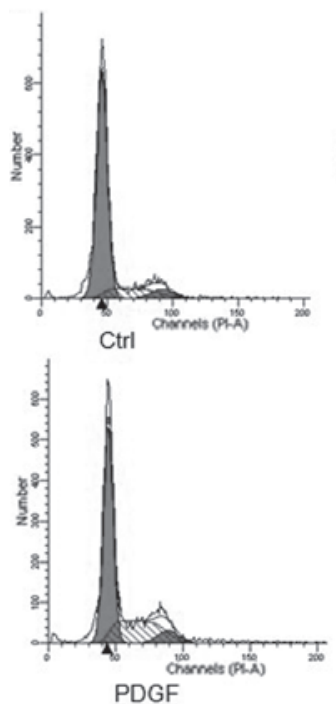

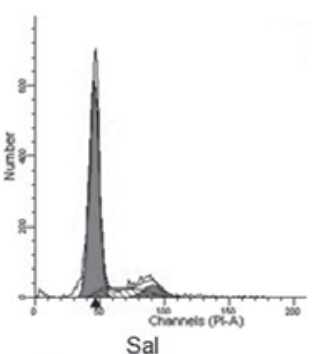

$\mathrm{Sal}$

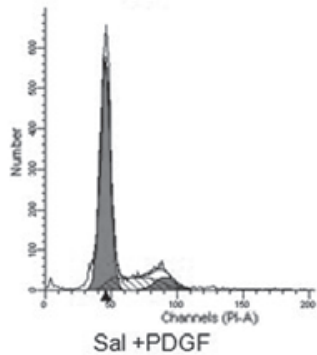

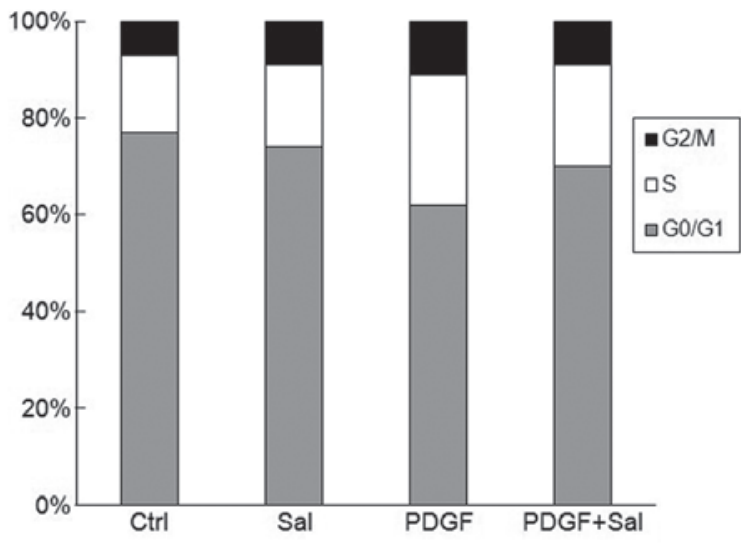

Figure 3. Effect of salidroside on PDGF-induced cell cycle progression. PASMCs were grown with salidroside (100 $\mu \mathrm{M})$ in the absence or presence of $20 \mathrm{ng} / \mathrm{ml}$ PDGF-BB for 24 h. (A) PI fluorescence in PASMCs as analyzed by flow cytometry. (B) Quantification of PASMCs in the G0/G1, S and G2/M phases, as determined by flow cytometric evaluation $(n=3)$. PDGF-BB, platelet-derived growth factor-BB; PASMCs, pulmonary artery smooth muscle cells; Ctrl, control; Sal, salidroside; PI, propidium iodide.

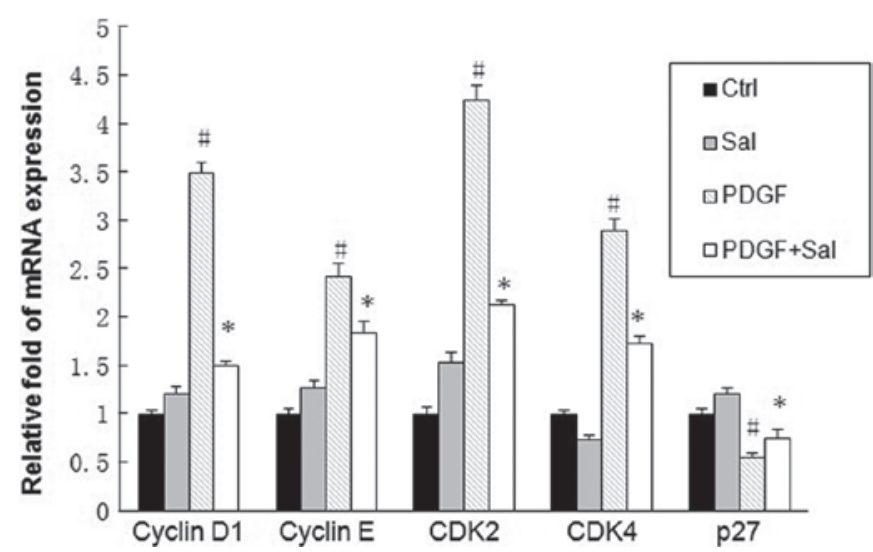

Figure 4. Effect of salidroside on the expression of multiple cell cycle regulatory genes in PASMCs. PASMCs were pretreated with salidroside $(100 \mu \mathrm{M})$ for $1 \mathrm{~h}$ prior to $24 \mathrm{~h}$ of $20 \mathrm{ng} / \mathrm{ml}$ PDGF-BB treatment and were analyzed for mRNA levels of multiple cell cycle regulatory genes. Experiments were performed in triplicate and repeated a minimum of three times. ${ }^{~} \mathrm{P}<0.05$ versus control; " $\mathrm{P}<0.05$ versus PDGF-treated cells; $\mathrm{n}=3$. CDK, cyclin-dependent kinase; PDGF-BB, platelet-derived growth factor-BB; PASMCs, pulmonary artery smooth muscle cells; Ctrl, control; Sal, salidroside.

the mRNA levels of cyclin D1, cyclin E, CDK2 and CDK4. Conversely, pretreatment with salidroside significantly suppressed the PDGF-induced upregulation of the studied genes (Fig. 4). The cyclin-CDK complexes formed in cell cycle progression are regulated by $\mathrm{CDK}$ inhibitors, such as p27, which leads to cell cycle arrest at the G1 and G1/S boundary (23). Pretreatment with salidroside upregulated the expression of $\mathrm{p} 27$.

Molecular mechanisms involved in the salidroside-induced inhibition of the proliferation of PASMCs. To explore the molecular mechanisms by which salidroside inhibits the proliferation of PASMCs, the effects of salidroside on MAPK and AKT/GSK3 $\beta$ signaling were examined. Significant acti-

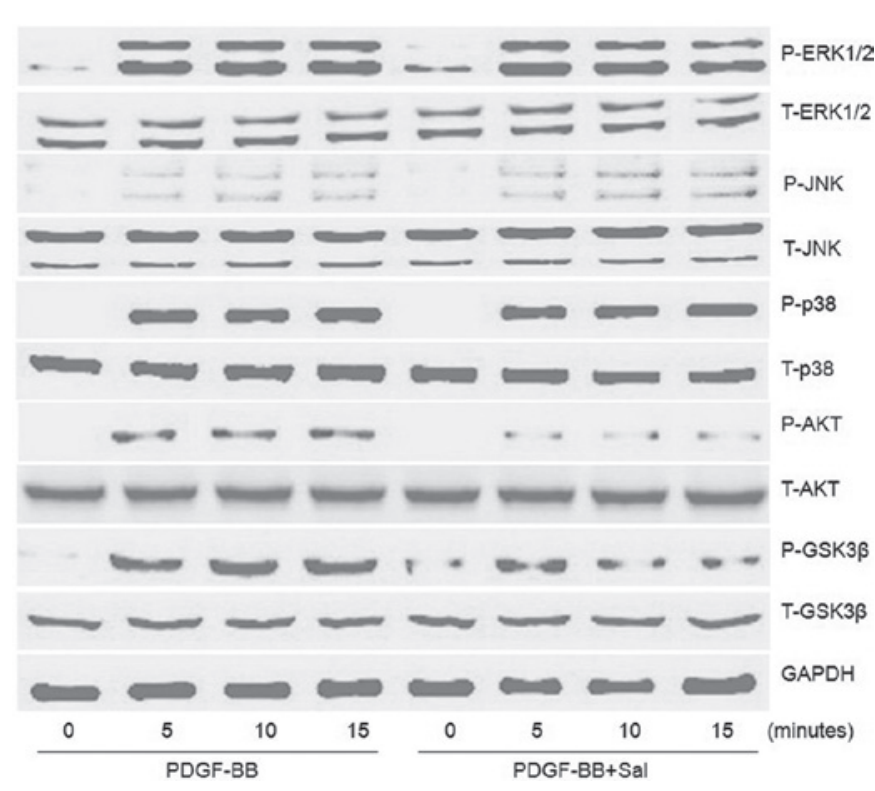

Figure 5. Effect of salidroside on the activation of signaling pathways in PDGF-BB-stimulated PASMCs. PASMCs were pretreated with salidroside $(100 \mu \mathrm{M})$ for $1 \mathrm{~h}$ prior to $20 \mathrm{ng} / \mathrm{ml}$ PDGF-BB treatment. The protein levels of P-ERK1/2, ERK1/2, P-JNK, JNK, P-p38, p38, P-AKT, AKT, P-GSK3 $\beta$ and GSK $3 \beta$ induced by PDGF-BB at 5,10 and 15 min were determined by western blot analysis. One representative image out of three independently performed experiments is shown. PDGF-BB, platelet-derived growth factor-BB; PASMCs, pulmonary artery smooth muscle cells; Sal, salidroside; ERK, extracellular signal-regulated kinase; JNK, c-Jun N-terminal kinase; GSK $3 \beta$, glycogen synthase kinase $3 \beta$; P-, phosphorylated.

vation of ERK1/2, p38, JNK and AKT/GSK3 $\beta$ was observed 5, 10 and 15 min after PDGF treatment, without affecting the total levels of these molecules (assessed by comparison with internal controls using western blotting) (Fig. 5). Salidroside significantly reduced the phosphorylation of AKT/GSK3 $\beta$, but did not exhibit any inhibitory effects on the phosphorylation of ERK1/2, p38 and JNK (Fig. 5). 


\section{Discussion}

The present study demonstrated that salidroside inhibits PDGF-induced PASMC proliferation and DNA synthesis in a dose- and time-dependent manner without cell cytotoxicity. It also showed that salidroside inhibits the cell cycle at G0/G1 to $\mathrm{S}$ phase through inhibition of the mRNA expression of cyclin D1, cyclin E, CDK2 and CDK4, as well as through an increase in the mRNA expression of p27, in PDGF-BB-stimulated PASMCs. These effects of salidroside on the proliferation of PASMCs were associated with inhibition of the AKT/ GSK3 $\beta$ pathway. These results suggest that salidroside may be a novel therapy for preventing pulmonary vascular remodeling diseases.

Abnormal proliferation of PASMCs leads to medial vascular lumen narrowing and vascular remodeling, which are critical to the development of PAH $(19,20)$. In the present study, it was demonstrated that salidroside inhibited PDGF-induced PASMC proliferation and DNA synthesis in a dose- and time-dependent manner without cell cytotoxicity. Cell proliferation is tightly regulated by the cell cycle, and salidroside has been demonstrated to cause cell cycle arrest in cancer cell lines $(16,18)$. For this reason, the effects of salidroside on the cell cycle in PDGF-BB-stimulated PASMCs were examined. Flow cytometric analysis results demonstrated that $100 \mu \mathrm{M}$ salidroside treatment for $24 \mathrm{~h}$ led to a significant increase in the number of cells in the G0/G1 phase and a reduction in the number of cells in the $S$ phase, without any significant effect on the number of cells in the G2/M phase. Taken together, these results indicate that salidroside targets a signaling transduction event evoked in the G0/G1-S boundary. Key factors of cell cycle regulation include the CDKs, which may be activated in a specific cell cycle phase by phosphorylation of the corresponding substrates, allowing for progression through the cell cycle. The CDKs are also dependent on cyclins, whose expression levels are associated with different cell cycle phases. Therefore, the degree of CDK activation is different for each stage of the cell cycle and plays a critical role in cell cycle regulation. The activity of CDKs may be inhibited by cell cycle inhibitory proteins (CKIs). CDK2 and CDK4 are known to form complexes with cyclin E and cyclin D1, which are essential for the mediation of cell cycle progression from the G0/G1 to $\mathrm{S}$ phases $(21,22)$. Another regulator controlling cell cycle progression is the CKI p27, which forms heterotrimeric complexes with cyclins and CDKs to inhibit their activity, such as cyclin D-CDK4 and cyclin E-CDK2 (23).

In this study, the expression of cell cycle regulatory genes in response to PDGF-BB in PASMCs was investigated. Salidroside reduced the PDGF-BB-induced mRNA expression of cyclin D1, cyclin E, CDK2 and CDK4. Consistent with these changes, the mRNA expression of p27 was increased by salidroside. These observations suggest that the antiproliferative activity of salidroside has a multifaceted effect on numerous target molecules critically involved in growth inhibition.

MAPK families, including ERKs, p38 and JNK, as well as the AKT pathway, play an important role in the regulation of cell proliferation. A previous study by our group demonstrated that PDGF-BB can stimulate the activation of ERK, JNK and p38, as well as the AKT/GSK3 $\beta$ pathway in VSMCs (24). In the present study, it was found that PDGF-BB can significantly stimulate the phosphorylation of ERK1/2, JNK, p38 and AKT/GSK $3 \beta$ in PASMCs. The PI3K/AKT signaling pathway is implicated in multiple cellular processes, including proliferation, differentiation, apoptosis and migration. AKT has numerous downstream targets, and GSK3 $\beta$ is one of its critical downstream molecules. Data from this study showed that salidroside inhibited the phosphorylation of AKT/GSK3 $\beta$ during PDGF-BB induction. However, this result contrasts those obtained by other studies, in which salidroside was reported to stimulate the AKT pathway $(25,26)$. Whether these differences are associated with different types of cells and different salidroside concentrations used in the studies remains to be elucidated. Cyclin D1 is regulated by GSK3 $\beta$, which can be inactivated by phosphorylation $(27,28)$. Activation of GSK3 $\beta$ has been found to regulate cyclin D1 export from the nucleus to the cytoplasm for proteolysis and thus decreases the expression of cyclin D1 (29). Furthermore, inhibition of the phosphorylation of the AKT/GSK3 $\beta$ signaling pathway has been shown to decrease the expression of cyclin D1 in cultured VSMCs (30). GSK3 $\beta$ inhibition has also been shown to decrease expression of the CKI p27 (31). Based on these results, it is possible that salidroside suppresses the proliferation of PASMCs through the AKT/GSK3 $\beta$ signaling pathway. The effect of salidroside on the activation of MAPKs, including ERK1/2, p38 and JNK, which are other important factors implicated in the PASMC proliferation induced by PDGF-BB, was also examined. Unlike its effect on the AKT/GSK3 $\beta$ pathway, salidroside failed to affect the PDGF-stimulated activation of ERK1/2, p38 and JNK. However, a previous study indicated that salidroside can inhibit the ERK, p38 and JNK signaling pathways in LPS-induced mastitis (15). It is possible that salidroside has different effects on the MAPK signaling pathways in different types of cells. These results indicate that the PDGF-BB-mediated activation of ERK, p38 and JNK may not be involved in the inhibitory effect of salidroside on the proliferation of PASMCs.

In conclusion, this study, to the best of our knowledge, demonstrated for the first time that salidroside inhibits the proliferation of PASMCs induced by PDGF-BB. Notably, this process appears to be associated with an inhibition of cyclin D1 expression and an increase in p27 accumulation through blockade of the AKT/GSK3 $\beta$ signaling pathway. The results of this study suggest that the use of salidroside may be feasible for therapies to treat pulmonary vascular remodeling diseases.

\section{References}

1. Humbert M, Sitbon $\mathrm{O}$ and Simonneau G: Treatment of pulmonary arterial hypertension. N Engl J Med 351: 1425-1436, 2004.

2. Sakao S, Tatsumi K and Voelkel NF: Reversible or irreversible remodeling in pulmonary arterial hypertension. Am J Respir Cell Mol Biol 43: 629-634, 2010.

3. Agbani EO, Coats P, Mills A and Wadsworth RM: Peroxynitrite stimulates pulmonary artery endothelial and smooth muscle cell proliferation: involvement of ERK and PKC. Pulm Pharmacol Ther 24: 100-109, 2011.

4. Luo C, Yi B, Bai L, et al: Suppression of Akt1 phosphorylation by adenoviral transfer of the PTEN gene inhibits hypoxia-induced proliferation of rat pulmonary arterial smooth muscle cells. Biochem Biophys Res Commun 397: 486-492, 2010.

5. Giese NA, Marijianowski MM, McCook O, et al: The role of alpha and beta platelet-derived growth factor receptor in the vascular response to injury in nonhuman primates. Arterioscler Thromb Vasc Biol 19: 900-909, 1999. 
6. Perros F, Montani D, Dorfmüller P, et al: Platelet-derived growth factor expression and function in idiopathic pulmonary arterial hypertension. Am J Respir Crit Care Med 178: 81-88, 2008.

7. Ogawa A, Nakamura K, Matsubara H, et al: Prednisolone inhibits proliferation of cultured pulmonary artery smooth muscle cells of patients with idiopathic pulmonary arterial hypertension. Circulation 112: 1806-1812, 2005.

8. Sanchez O, Marcos E, Perros F, et al: Role of endothelium-derived $\mathrm{CC}$ chemokine ligand 2 in idiopathic pulmonary arterial hypertension. Am J Respir Crit Care Med 176: 1041-1047, 2007.

9. Berg JT, Breen EC, Fu Z, et al: Alveolar hypoxia increases gene expression of extracellular matrix proteins and platelet-derived growth factor-B in lung parenchyma. Am J Respir Crit Care Med 158: 1920-1928, 1998

10. Díaz Lanza AM, Abad Martínez MJ,Fernández Matellano L, et al: Lignan and phenylpropanoid glycosides from Phillyrea latifolia and their in vitro anti-inflammatory activity. Planta Med 67: 219-223, 2001.

11. De Sanctis R, De Bellis R, Scesa C, et al: In vitro protective effect of Rhodiola rosea extract against hypochlorous acid-induced oxidative damage in human erythrocytes. Biofactors 20: 147-159, 2004.

12. Mattioli L and Perfumi M: Rhodiola rosea L. extract reduces stress- and CRF-induced anorexia in rats. J Psychopharmacol 21 742-750, 2007

13. Ming DS, Hillhouse BJ, Guns ES, et al: Bioactive compounds from Rhodiola rosea (Crassulaceae). Phytother Res 19: 740-743, 2005.

14. Kanupriya, Prasad D, Sai Ram M, et al: Cytoprotective and antioxidant activity of Rhodiola imbricata against tert-butyl hydroperoxide induced oxidative injury in U-937 human macrophages. Mol Cell Biochem 275: 1-6, 2005.

15. Li D, Fu Y, Zhang W, et al: Salidroside attenuates inflammatory responses by suppressing nuclear factor- $\mathrm{\kappa B}$ and mitogen activated protein kinases activation in lipopolysaccharide-induced mastitis in mice. Inflamm Res 62: 9-15, 2013.

16. Sun C, Wang Z, Zheng Q and Zhang H: Salidroside inhibits migration and invasion of human fibrosarcoma HT1080 cells. Phytomedicine 19: 355-363, 2012.

17. Yin D, Yao W, Chen S, et al: Salidroside, the main active compound of Rhodiola plants, inhibits high glucose-induced mesangial cell proliferation. Planta Med 75: 1191-1195, 2009.
18. Hu X,Zhang X, Qiu S, et al: Salidroside induces cell-cycle arrest and apoptosis in human breast cancer cells. Biochem Biophys Res Commun 398: 62-67, 2010.

19. Pietra GG, Capron F, Stewart S, et al: Pathologic assessment of vasculopathies in pulmonary hypertension. Am Coll Cardiol 43 (12 Suppl S): 25S-32S, 2004.

20. Rabinovitch M: The mouse through the looking glass: a new door into the pathophysiology of pulmonary hypertension. Circ Res 94: 1001-1004, 2004

21. Jirawatnotai S, Aziyu A, Osmundson EC, et al: Cdk4 is indispensable for postnatal proliferation of the anterior pituitary. J Biol Chem.279: 51100-51106, 2004.

22. Martín A, Odajima J, Hunt SL, et al: Cdk2 is dispensable for cell cycle inhibition and tumor suppression mediated by p27(Kip1) and p21(Cip1). Cancer Cell 7: 591-598, 2005.

23. Abukhdeir AM and Park BH: P21 and p27: roles in carcinogenesis and drug resistance. Expert Rev Mol Med 10: e19, 2008.

24. Guan H, Chen C, Zhu L, et al: Indole-3-carbinol blocks platelet-derived growth factor-stimulated vascular smooth muscle cell function and reduces neointima formation in vivo. J Nutr Biochem 24: 62-69, 2013.

25. Zhang L, Ding W, Sun H, et al: Salidroside protects PC12 cells from $\mathrm{MPP}^{+}$-induced apoptosis via activation of the PI3K/Akt pathway. Food Chem Toxicol 50: 2591-2597, 2012.

26. Zhu Y, Shi YP, Wu D, et al: Salidroside protects against hydrogen peroxide-induced injury in cardiac H9c2 cells via PI3K-Akt dependent pathway. DNA Cell Biol 30: 809-819, 2011.

27. Diehl JA, Cheng M, Roussel MF and Sherr CJ: Glycogen synthase kinase-3beta regulates cyclin D1 proteolysis and subcellular localization. Genes Dev 12: 3499-3511, 1998.

28. Yu XJ, Han QB, Wen ZS, et al: Gambogenic acid induces G1 arrest via GSK3 $\beta$-dependent cyclin D1 degradation and triggers autophagy in lung cancer cells. Cancer Lett 322: 185-194, 2012.

29. Malumbres M and Barbacid M: To cycle or not to cycle: a critical decision in cancer. Nat Rev Cancer 1: 222-231, 2001.

30. Yin M, Tian S, Huang X, et al: Role and mechanism of tissue plasminogen activator in venous wall fibrosis remodeling after deep venous thrombosis via the glycogen synthase kinase- 3 beta signaling pathway. J Surg Res 184: 1182-1195, 2013.

31. Tseng AS, Engel FB and Keating MT: The GSK-3 inhibitor BIO promotes proliferation in mammalian cardiomyocytes. Chem Biol 13: 957-963, 2006. 